\section{SPATIALLY ORGANIZED MULTICELLULAR IMMUNE HUBS IN MMRD AND MMRP COLORECTAL CANCER}

'Jonathan Chen*, ${ }^{2}$ Karin Pelka, ${ }^{2}$ Matan Hofree, ${ }^{3}$ Marios Giannakis, ${ }^{1}$ Genevieve Boland, ${ }^{3}$ Andrew Aguirre, ${ }^{4}$ Ana Anderson, ${ }^{2}$ Orit Rozenblatt-Rosen, ${ }^{2}$ Aviv Regev, ${ }^{1}$ Nir Hacohen. ${ }^{1}$ Massachusetts General Hospital, Cambridgee, MA, United States; ${ }^{2}$ Broad Institute, Cambridge, MA, United States; ${ }^{3}$ Dana-Farber Cancer Institute, Boston, MA, United States; ${ }^{4}$ Hanvard Medical School, Boston, MA, United States

Background Immune responses to cancer are highly variable, with DNA mismatch repair-deficient (MMRd) tumors exhibiting more anti-tumor immunity than mismatch repair-proficient (MMRp) tumors. Almost all tumors are infiltrated with immune cells, but the types of immune responses and their effects on tumor growth, metastasis and death, vary greatly between different cancers and individual tumors. Which of the numerous cell subsets in a tumor contribute to the response, how their interactions are regulated, and how they are spatially organized within tumors remains poorly understood.

Methods To understand the rules governing these varied responses, we transcriptionally profiled 371,223 single cells from colorectal tumors and adjacent normal tissues of 28 MMRp and 34 MMRd treatment-naive patients. We developed a systematic approach to discover cell types, their underlying gene programs, and cellular communities based on single cell RNA-seq (scRNAseq) profiles and applied it to study the distinguishing features of human MMRd and MMRp colorectal cancer. Cellular communities discovered from this analysis were spatially mapped in tissue sections using multiplex RNA in situ hybridization microscopy.

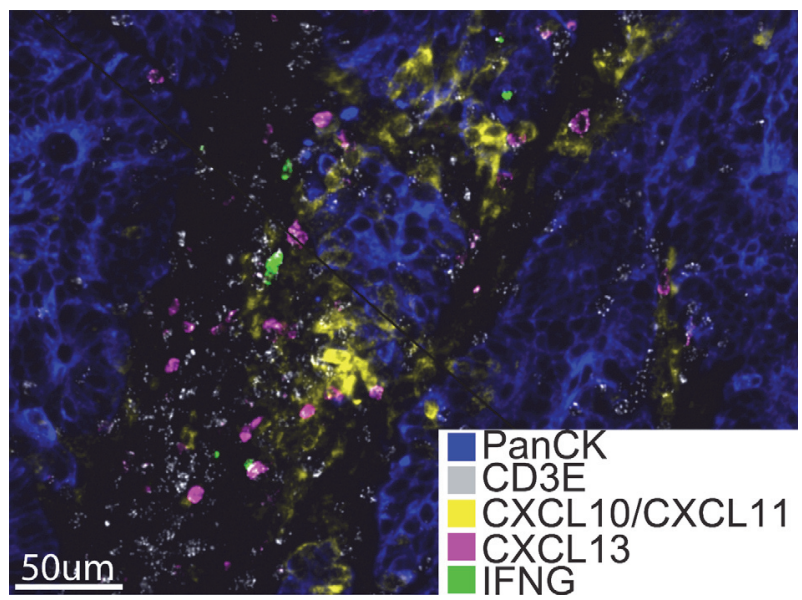

Abstract 641 Figure 1 A coordinated network of CXCL13+ T cells with myeloid and malignant cells expressing ISGs. Image shows a portion of formalin-fixed paraffin-embedded tissue from an MMRd CRC specimen stained with multiplex RNA ISH / IF for PanCK-IF, CD3E-ISH, CXCL10/CXCL11-ISH, CXCL13-ISH, and IFNG-ISH. Note IFNG+ and CXCL13+ cells in proximity to cells expressing the chemokines CXCL10/ CXCL11

Results To understand the basis for differential immune responses in CRC, we first determined and compared the immune cell composition of MMRd and MMRp CRC and normal colon tissue, finding dramatic remodeling between tumor and normal tissue and between MMRd and MMRp tumors, particularly within the myeloid, T cell, and stromal compartments. Among the clusters enriched in MMRd tumors were activated CXCL13+ CD8 T cells. Importantly, gene program co-variation analysis revealed multicellular networks. We discovered a myeloid cell-attracting hub at the tumor-luminal interface associated with tissue damage, and an MMRdenriched immune hub within the tumor, with activated IFNG + and CXCL13 $+\mathrm{T}$ cells together with malignant and myeloid cells expressing T-cell-attracting chemokines (figure 1).

Conclusions Our study provides a rich dataset of cellular states, gene programs and their transformations in tumors across a relatively large cohort of patients with colorectal cancer. Our predictions of several multicellular hubs based on covariation of gene expression programs, and subsequent spatial localization of two major immune-malignant hubs, organizes a large set of cell states and programs into a smaller number of coordinated networks of cells and processes. Understanding the molecular mechanisms underlying these hubs, and studying their temporal and spatial regulation upon treatment will be critical for advancing cancer therapy.

Ethics Approval This study was approved by the DF-HCC institutional review board (protocols 03-189 and 02-240).

http://dx.doi.org/10.1136/jitc-2021-SITC2021.641 